\title{
Structural and functional characterization of bifunctional enzyme encoded by ribBX gene in riboflavin biosynthesis pathway of Helicobacter pylori 26695.
}

\author{
$\underline{\text { Ruchi Gautam }}^{1}$, Karthikeyan Subramanian ${ }^{1}$ \\ ${ }^{1}$ Protein science and engineering division, CSIR-Institute of Microbial Technology, Sector 39 A, \\ Chandigarh 160036, India \\ E-mail : ruchigautam@imtech.res.in
}

\begin{abstract}
Riboflavin (vitamin B2) is the precursor of flavin mononucleotide (FMN) and flavin adenine dinucleotide (FAD), which are essential cofactors for intracellular redox reactions and play important role in cellular physiology including iron acquisition, extracellular respiration, and interspecies interactions. Microorganisms synthesize flavin to fulfill their nutritional requirements. Flavins are important for assimilatory iron reduction in Campylobacter jejuni, Helicobacter pylori. Genes involved in riboflavin pathway are found to be essential in Helicobacter species and enzymes of this pathway can be potential drug target against cancer caused by Helicobacter sps. Phylogenetic analysis revealed that this gene, renamed ribBX misannotated as ribBA because putative GTP cyclohydrolase II domain lacks catalytic activity. The persistence of ribBX gene in divergent bacterial genomes shows that it has evolved a function which leads a selective advantage to the host.

In Helicobacter pylori 26695, we characterized N-terminal domain of ribBX as DHBPS. Gel filtration and crystal structure reveals that $\mathrm{N}$-terminal domain exists as monomer. We attempted structural characterization of bifunctional enzyme encoded by ribBX gene from other homologous organisms. A partial structure of low resolution (4.2 $\AA$ ) shows that over all fold of hribBX enzyme remains same with bifunctional DHBPS/ GCHII but lacks GCHII function. This natural C-terminal truncation leads to loss of function or different function of $\mathrm{C}$-terminal domain.
\end{abstract}

